Review

\title{
Some remarks on Colloid Stability: Selected Examples Taken from the Milk Chain for Food Prepares
}

\author{
Camillo La Mesa 1,* and Gianfranco Risuleo $2, \dagger$ \\ 1 Department of Chemistry, Cannizzaro Building, La Sapienza University, Piazzale Aldo Moro 5, \\ 00185 Rome, Italy. \\ * Corresponding Author \\ Correspondence: camillo.lamesa@uniroma1.it \\ 2 Department of Biology and Biotechnology Charles Darwin, La Sapienza University, Piazzale \\ Aldo Moro 5, 00185 Rome, Italy. \\ Correspondence: gianfranco.risuleo@uniroma1.it or gianfranco.risuleo@gmail.com \\ $\dagger$ Retired
}

\begin{abstract}
Different forces play a key-role in the stability of food colloid dispersions. Focus is here on those controlling attraction and/or repulsion, which concur to stabilization, phase separation, coagulation, and are quite evident in water-based systems. Combination of attractive and repulsive forces favors, or hinders, the association of colloid entities; such processes are often met in food technology. The above processes depend on the forces at work, and on colloid concentration in the medium (i.e. on inter-particle distance). Worked examples deals with milk manipulation procedures, ending in cheese formation. The whole milk-working sequence is controlled by the combination of forces leading to aggregation and phase separation of casein and other milk components. Thereafter, one gets either fresh, for prompt consumption, or aged cheeses. The combination of attractive (van der Waals, $v d W$, and depletion) with repulsive (double layer, $D L$, but also with steric) forces results in the dominance of aggregation versus dispersion modes in all steps of milk transformation, which depend on the distance among colloid particles, on the amplitude of the mentioned forces, and on their decay length. The combined role of double layer and van der Waals $(v d W)$ forces is at the basis of the DLVO theory on colloid stability, which is properly modified when these forces overlap with steric stabilization and, eventually, with depletion. Steric effects are dispersive, depletion ones favor colloid nucleation in a single phase. The milk manipulation chain is a worked example of the intriguing association features controlled by the mentioned forces (and of ancillary ones, as well), and indicates which forces favor the formation of products such as Parmesan or Mozzarella cheese, but are not alien to the preparation of many other dairy products.
\end{abstract}

Keywords: Attractive and Repulsive forces, Debye's screening length (1/k), Poisson-Boltzmann $(P B)$ equation, food colloids, coagulation, stabilizers, lipids, polymers, electrolytes.

\subsection{Introduction}

The cohesive forces occurring among entities in any physical body become less relevant on passing from solid to liquid and gaseous form. Strong forces permit vibrations around the equilibrium position, moderate to strong translational motions (in liquids), weak ones free and unrestricted motions, as in gases. The same also holds in colloids, be they solid, liquid or bubbles. Colloids move as a whole kinetic entity in a fluid: diffusion in water-based media is a typical example. The colloid state, thus, deals with dispersion of solids, droplets, or bubbles in another phase. We may observe reciprocal dispersions of solids, as in opals (remaining as such for indefinitely long times), of solids in liquids or gases, liquids or bubbles in liquids, and so forth, Table I. Given the peculiar nature of liquids, colloid stabilization is important in systems where the dispersant is such.

Stabilized colloid particles behave as planets, and sense different forces. It is not surprising, therefore, that an eminent scientist (Nobel Award in 1983) put in evidence the similarities between 
Table I. Possible Colloid Dispersion Modes.

\begin{tabular}{cccc}
\hline $\begin{array}{c}\text { State of matter } \\
\text { Ist component } \\
\text { (dispersant) }\end{array}$ & $\begin{array}{c}\text { the IInd component } \\
\text { is a solid } \\
\text { (dispersed) }\end{array}$ & $\begin{array}{c}\text { the IInd component } \\
\text { is a liquid } \\
\text { (dispersed) }\end{array}$ & $\begin{array}{c}\text { the IInd component } \\
\text { is a gas } \\
\text { (dispersed) }\end{array}$ \\
\hline Solid & opals & bitumens & foams \\
\hline Liquid & muds & emulsions* & no \\
\hline Gas & smokes & aerosols & \\
\hline
\end{tabular}

the colloidal domain and stellar physics [1]. Despite that, most humans consider colloids as "magical" entities, whose nature appears unfamiliar as the gravitational laws. We clarify below some quintessential points of colloid stability, by the same methodological approach, i.e. considering the action of different forces concurring to these processes. In words, we deal with the same mental attitude that is used to account for the motion of planets.

Aim of this contribution is to induce a correct scientific attitude, to avoid the consolidation of absurd opinions. The ironical sentence below is an example of the behavior to be avoided. "Reporting on the motions of earth and planets, Galileo treated the catholic church with the same wickedness that a classmate used against my beliefs. He told me "Santa Claus does not exist, reindeer-driven sleighs neither". How different I was from him! Should granny have told me that donkeys fly, I should have never have replied they do not, but, presumably, I would have added that horses, mules, ponies, the seven dwarves, the twelve apostles and our archbishop

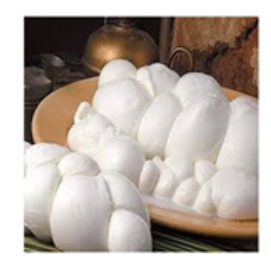

A

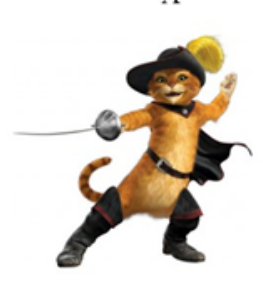

$\mathrm{C}$

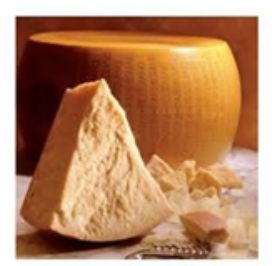

B

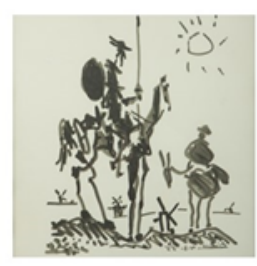

D

Figure 1. A: "braided" fresh mozzarella from buffalo cheese. Produced mainly in central-southern Italy, is possibly appreciated all over the world. B: Parmesan cheese shape and wedge. The most select parmesan cheese may have undergone up to 36 months of aging in controlled atmosphere. The standard weight of a matured parmesan shape is about $40 \mathrm{~kg}$, for which about 550 liters of cow milk are required. C The Booted Cat (Le Chat Botté) the main character of the fable by the French novelist Charles Perrault (Paris, 1628-1703). D: Sketch by Pablo Picasso depicting the Knight Don Quixote and the faithful squire Sancho possibly mumbling over the opportunity to storm the windmill. 
(despite his modernistic attitudes) do that. And to render granny happier I should have told her that in Paradise she will have met Pinocchio and Geppetto, the Booted Cat (Le Chat Bottee), the Red Feathered Young Teacher, the harmonious Smith and Queen Margret, angels, saints and other canonically expected guests" [2]. We try to persuade readers not to behave as the mad character depicted above. Figure 1 shows some items discussed in the following, and some personnel too.

Focus here is on food colloids, such as milk, mayonnaise, chocolate, and juices. These are sometimes obtained with the help of mechanical energy, crumbling the raw products and giving to these dispersions the desired shapes and forms, by imparting energy, later dissipated in form of heat [3]. The optimization of food colloids is of fundamental interest, due to the growing demand for a wide availability of staple foods, which are characterized by high quality, and nutritious capacity. Raw matter quality, attention to manipulation and long-term stabilization are relevant starting points [4]. Below, we focus on the steps to be followed in the food manipulation chain and on the forces controlling each of them. That's why food technicians tend to optimize the following manipulation stages:

a) collecting raw matter;

b) operating in such a way to get the desired products of a given food chain in substantial amounts;

c) obtaining the final products through maturing and storage, if these steps are required.

Cooking, drying, salting, smoking and storage procedures belong to the human knowledge since many thousand years [5-7]. Preparations as anchovies in paste [8], stock cubes, and cheese $[9,10]$, are pertinent examples; many among these products use stabilizers during the manipulation stages, and all are colloidal in nature, or are such during some manipulation steps.

We deal with fluid, semi-fluid matrices, creams and pastes; in other words, with "soft matter" foods. To make such preparations reliable, nutritionally safe, with "permanent" macroscopic appearance, homogeneity upon aging, and retainment of adequate properties throughout different batches and seasons must be granted. Food preparations are also optimized from a chemical and biological viewpoint, to fulfill the due health requirements. According to the rules, and good sense, any colloid-based food must be stable and retain its peculiarities and taste for long times. There is also urgent need that storage does not require conditions hardly at hand in disadvantaged situations, when, for instance, low-temperature chains cannot be guaranteed. Old-fashioned preparations, which are costly niche products, may give high quality matter, but operate in small to medium scale, with drawbacks due to their costs, retailing and durability.

Food manipulation gives creams, fluids, pastes, sauces, etc., which are stabilized by adding salts, lipids, some proteins, or polysaccharides [11-14]. Stabilizers are preferentially taste-neutral, biocompatible, and not too much expensive. They should be get from natural sources in large amounts, and, compatibly, from the same materials as the products to be stabilized. Food colloids have rheological properties allowing them to flow or spread under moderate shear conditions. In addition, their nutritional quality must fulfill the standards required from national/international procedural guidelines [15].

We indicate below which forces are mainly responsible for food colloids stability and apply pertinent information to the case of cheese. This largely consumed commodity (there are several hundred cheese types, perhaps) undergoes many transformations from raw milk. The latter, a waterbased bio-colloid, contains fats, but also proteins, (about $82 \%$ is made of different casein types and the remaining serum or whey proteins) [16], sugars (mostly lactose), salts, etc. Fat content depends on mammal type, Table II. Similar considerations hold for protein, sugar, and salt content. Goat milk, for instance, has low amounts of calcium and phosphate, and is enriched with their salts [17].

\subsection{Forces in Action and Species Responsible for Stabilization}

Different chemical, biochemical, and physico-chemical properties characterize the most common features of products and stabilizers used in the food industry. Among many forces at work, surface adsorption is 
Table II. Average fat matter percent in fresh milk of some mammal ruminants.

\begin{tabular}{ccc}
\hline Mammal Ruminant & Country & Fat \% in fresh milk \\
\hline Jersey cow & United Kingdom & $5,2 \%$ \\
\hline Zebu & India, Madagascar, Sri Lanka & $4,7 \%$ \\
\hline Brown Swiss cow & Switzerland & $4,0 \%$ \\
\hline Holstein Friesian cow & Netherlands, FRG, Denmark & $3,6 \%$ \\
\hline Neapolitan Buffalo & Italy & $8,3 \%$ \\
\hline Spanish sheep & Spain, Italy, Greece & $5,6 \%$ \\
\hline Merinos-modified sheep & Spain, Australia & $5,3 \%$ \\
\hline Appenzell goat & Switzerland & $4,6 \%$ \\
\hline Manchega goat & Spain, France & $4,8 \%$ \\
\hline & & \\
\hline & &
\end{tabular}

N.B. Data refer to average values inferred from different sources.

strictly necessary, since stabilization is almost always concomitant to interface saturation [18]. It is immaterial here if such processes arises by covalent or non-covalent polymer binding. Adsorption also implies wrapping [19], steric [20], osmotic [21], electrostatic effects [22], and combinations thereof [23]. It is hardly ascertained "a priori" if stabilization results from the combination of more contributions; think to the role of $k$-carragenans [24], or lipids [25], as stabilizers! The former are linear sulfated polysaccharides obtained seaweeds (by Chondrus crispus algae, also known with the vulgar name of Irish moss). They have been in use since the XVI century, but were known in China as early as the $6^{\text {th }}$ century BCE. Their main application is in dairy and meat products for their ability to establish strong bonds with food proteins [26]. Carragenans are vegetarian and vegan alternatives to gelatin and are also used to replace the latter in confectionery and ice creams $[27,28]$.

We mention below the forces responsible for the making and stability of cheese from the milk food chain. Among them surface adsorption, van der Waals $(v d W)$, steric and electrostatic forces are worth to be considered; others may be also present. We start with surface adsorption, somehow connected to steric stabilization; in fact, adsorption is a prerequisite for steric effects to occur. Later, some generalities on electrostatic and van der Waals forces will be given. The rationale inherent to the above sequence is evident. We stress again that steric effects arise if polymer surface adsorption has occurred, and are ancillary to it, exactly as Sancho Panza reluctantly follows the noble knight Don Quixote.

\subsection{Surface properties}

Adsorption onto colloids occurs through surface binding of stabilizers. These resulting forces are similar to those allowing surfactants to adsorb at fluid surfaces. Surface tension is due to the gain associated to the transfer of chemicals from the bulk to an interface; it is expressed by Gibbs isotherm, linking the adsorption energy, $G_{a d s}$, to surface tension, $\gamma$, and differential area, $d A$, as

$$
D G_{a d s}=\gamma d A
$$


The above relation is at the basis of surfactant action, mostly as to the adsorption of surfactants and lipids at air-liquid interfaces. The behavior of surfactants and lipids is well known: interested reader in this particular point may refer to classical compilations [29-31]. The equation applies to all substances adsorbing at interfaces, including protein adsorption on hydrophilic/hydrophobic surfaces, as they do in foams, and emulsions. Old-dated efforts to determine the molecular weight of lysozyme used surface tension methods (with appreciable results, indeed) [32]. Remind also that protein adsorption on nanoparticles to be used in biomedicine is detrimental and can be avoided by adding polymers, such as PEO, that preferentially adsorb thereon, thus reducing biopolymer adsorption [33].

We consider in what follows the surface behavior of stabilizers and bio-polymers, and assume that if the surface tension of their aqueous mixtures is lower than that of the solvent, then polymers adsorb. The surface tension, $\gamma$, of two-components systems (and not only them, perhaps) depends on composition, nature of adsorbed species, Figure 2. Temperature, conversely, makes possible to change surface composition from a polymer-rich to a polymer-depleted state, if such behavior holds true, Figure 3.

Stabilizers adsorb onto colloids thanks to surface tension. Such energy contribution is due to the transfer of chemicals from the bulk to interfaces and is expressed by Gibbs adsorption isotherm. If the surface tension of a polymer solution is lower than that of the solvent, the first adsorbs on the particle surface. For flat and fluid interfaces the Gibbs equation is defined as

$$
d \gamma=-\Gamma_{2} d \mu_{2}=-\Gamma_{2} R T d \ln a_{2}
$$

where $\mathrm{d} \gamma$ is the differential surface tension with respect to the solute, $\Gamma_{2}$ its excess concentration per unit area, $\mu_{2}$ the chemical potential of the solute, and $a_{2}$ the solute activity.

$E q$. (2) indicates that the differential of $\gamma$ is inversely proportional to the "partial molal area" $\left(1 / \Gamma_{2}\right)$ of the solute. The latter depends on the solute activity, $a_{2}$, and vanishes if there is no room for adsorption; that is, when the surface is saturated by the solute. Originally intended for flat fluid surfaces, Eq. (2) also applies to drops and emulsions. Complications arise if stabilizers adsorb onto porous solids [34]. In the above eventuality, the configuration of a flexible polymer interacting with a surface somehow depends on $T$, so that the given polymer is adsorbed, or repelled. Theoretical studies consider polymer adsorption on either smooth and porous solid surfaces $[35,36]$. Some remarks on this point are outlined in the forthcoming section.

\subsection{Steric Stabilization}

Such contribution is hardly understood on primitive grounds. In some sense, the stabilization operates in analogy to the action of a surface-anchored spring exerting a repulsive force per unit area. It is known that the structure and properties of long polymer chains, grafted or colloid-bound, control steric effects. Steric stability is considered entropy-controlled; indeed, it can be enthalpy- or entropy-driven [37]. It contains many other terms, as osmotic, excluded volume, conformational, electrostatic, and combinations. Due to the lack of a univocal theory, current models in the field use molecular dynamics, or other types of simulation procedures.

To account for steric stability we consider flat and parallel surfaces at well defined distances apart; in between, polymer and solvent are present in due amounts. Adsorption and spreading take place on both surfaces, until they are saturated by the given polymer. Expansion, compression, mixing with the solvent, and interpenetration stages continue to take place until full equilibrium conditions are attained. We consider forces acting among flat surfaces, Figure 4. Such behavior looks like to what we observe from surface forces apparatuses [38]. 


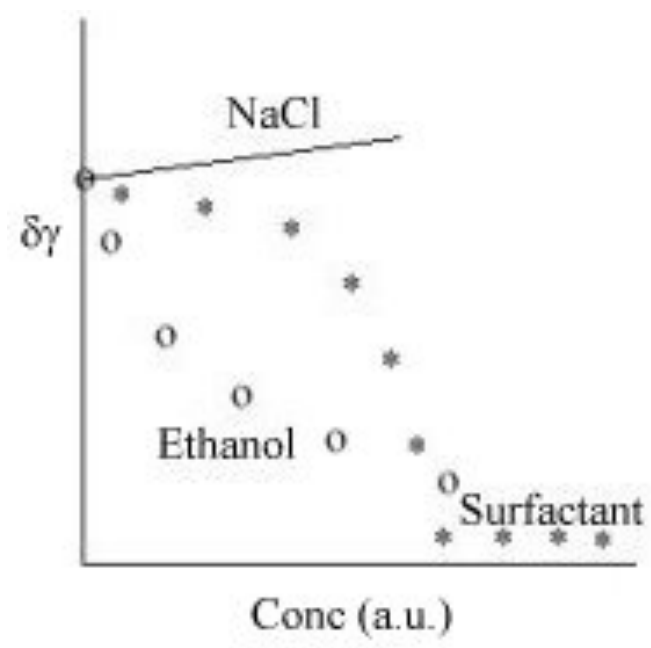

Figure 2. Variation of the surface tension of aqueous mixtures, $d \gamma$, as a function of a generic composition scale, in arbitrary units. Data refer to $\mathrm{NaCl}$, full line, ethanol, empty circles, and a surfactant. $\mathrm{NaCl}$ increases $\gamma$, i.e. $\mathrm{d} \gamma / \mathrm{dc}>0$, the other two species decrease it $(\mathrm{d} \gamma / \mathrm{dc}<0)$. Above a critical threshold, termed $c m c$, surface active species saturate the air-solution interphase and $\mathrm{d} \gamma$ remains nearly constant.

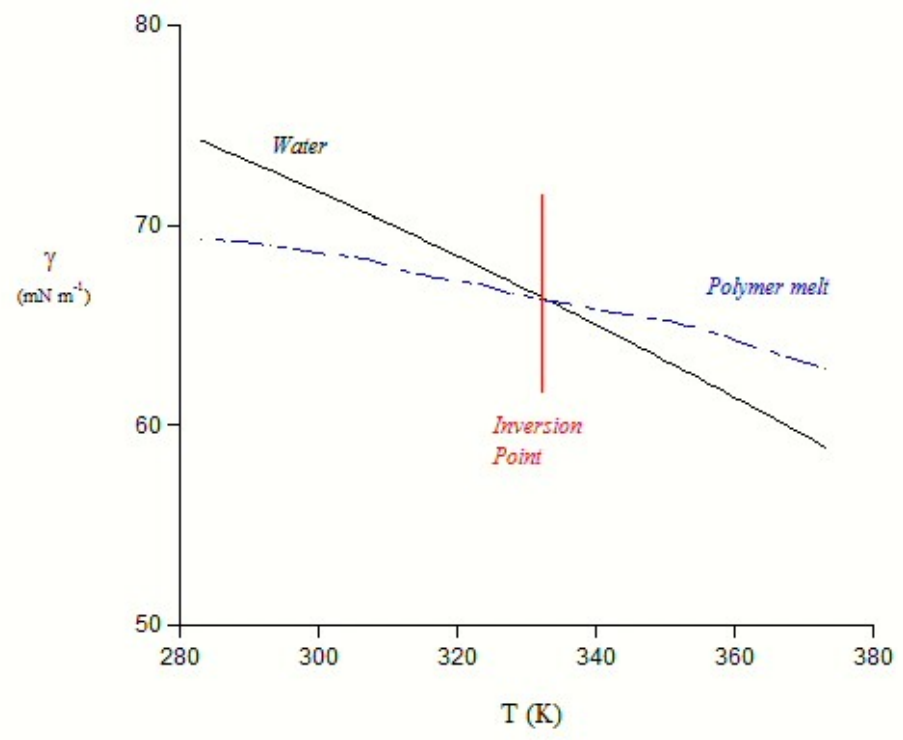

Figure 3. Surface tension of a water/polymer mixture, $\gamma$, as a function of temperature, in $\mathrm{K}$. The vertical line in the centre of figure indicates the inversion temperature, $T_{i n v}$, below which polymer is surface-depleted. On the right of the "inversion point" the polymer prefers the surface phase compared to the bulk one. If a solid is present, therefore, surface stabilization and polymer adsorption begin from this point onward.

Steric stabilization is evaluated by mean-field theories based on the interactions between two plates covered with densely grafted polymer chains; the concept "dense" is, perhaps, elusive. The interaction potential depends on the polymerization degree, and increases with it, on the stiffness of stabilizing chains, the solvent quality and on the number density of adsorbed chains. Steric stabilization, hereafter indicated as $\Xi$, depends on inter-particle attractive energy, $E_{\text {att }}$, on the amount of 
grafted polymer, $\sigma_{\text {Polym }}\left(C_{\text {polym }} / A_{\text {part }}\right)$, and on deviations from $\theta$-temperature conditions, $d \theta$. More refined approaches are at hand, but we better use here a primitive model, written as

$$
\Xi=f\left(E_{\text {att }}, \sigma_{\text {Polym }}, d \theta\right)
$$

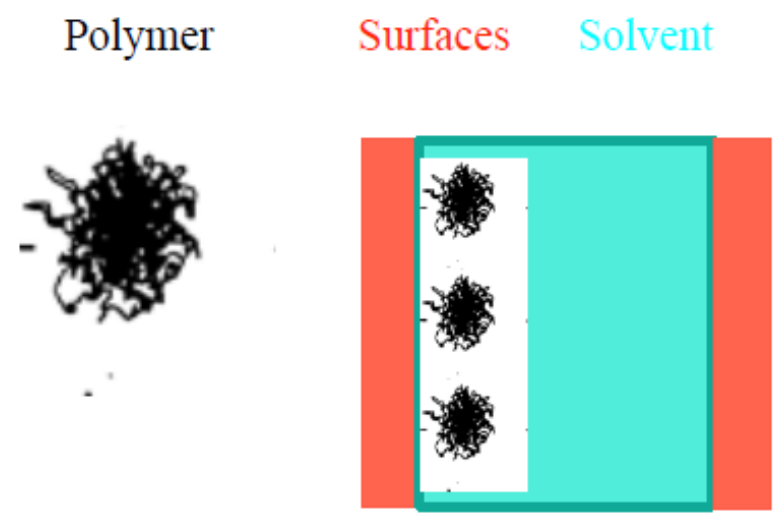

Distance, $\mathrm{X}$

Figure 4. Covered surfaces, in red, at a distance $X$ apart, with polymer and solvent in between. Polymers mix with the solvent, light blue; thus, surfaces saturate. Compression of solvated polymer films occurs and interpenetration takes place. (N.B. Polymer chains are not in direct contact with the surface, as they should be.) Each step has its own energy and the final stabilization is the sum of all these terms. The distance among the surfaces is, by definition, much higher than the average polymer size.

The minimum of $\Xi$ in the $E_{a t t}, \sigma, \theta$ space implies thermodynamic stability. For weak attractions, curves calculated by $E q$. (3) display a secondary minimum. If $E_{\text {att }}$ is lower that all other terms, thermodynamic stability conditions are met. For strong inter-particle attraction, in presence of a good solvent for the polymer chains, the potential displays primary and secondary minima, separated each other by a maximum. The stability, in the latter case, is kinetic in character [39]. Other effects are concomitant to steric stabilization, as osmotic, depletion (due to concentration gradients sensed by surface-partitioned polymer chains between two particles), and electrostatic (it refers to charged polymers). Sometimes it is necessary to reduce steric stability and favor nucleation. A pertinent case is due to the action of rennet, an enzyme cutting proteins facing outward casein clusters, and facilitating the cheese seeds onset.

\subsection{Electrostatic contributions}

Electrostatics influences food stabilization and it is, thus, required knowing some aspects of colloid stability expressed in terms of $D L V O$ theory [40,41], which combines $v d W$ and double layer, $D L$, forces. The resulting energy is due to the overlapping of such forces; the former is always at- 
tractive, the latter repulsive. Refinements and implementations of the theory are available [42-45]. $D L V O$ theory explains why colloids disperse, attract or coagulate, depending on the experimental conditions.

Combination of these forces controls the interactions. Stability can revert to instability upon very tiny changes in the control variables, such as salts content, $p H$ or screening the surface charge density in some other way. Similarly charged surfaces undergo to long-range repulsions, and the barriers keeping them apart can be some $K_{B} T$ units high. If the electrolyte content increases, a secondary minimum occurs. In such conditions, $\sigma$ (the surface charge density) approaches zero, the repulsive forces minimize, and attractive terms operating in the overlapping range dominate, Figure 5. Colloids are characterized by a given size and electrical surface potential. Uncharged colloids coagulate, whereas surface charges density avoid it. Stabilizers impart a "permanent" surface charge density. Consider two surfaces as specular images each of the other, both with known $|\sigma|$ value. In consequence of that, surfaces repel. Physically, the behavior is the same as that between two planes bearing equal $\Psi$ values. Potentials decay as

$$
\Psi(x)=\Psi^{\circ} \exp -k D
$$

Repulsion depends on the potential, $|\Psi|$, which exerts a long distance effect and scales with $k D . D$ is the distance among particles and $1 / k$ is Debye's screening length (formally, the distance at which the potential decays, as reported below). Thus, $D$ is the distance from a virtual charged surface of potential $\Psi^{\circ}$, Figure 6. Equation (4) refers to the interaction between two surfaces (a real and an ima ginary one). The decay of potential in between is written as

$$
\nabla^{2} \Psi=d^{2} \Psi / d x^{2}+d^{2} \Psi / d y^{2}+d^{2} \Psi / d z^{2}=-\left(\varrho / \varepsilon \varepsilon^{\circ}\right)
$$

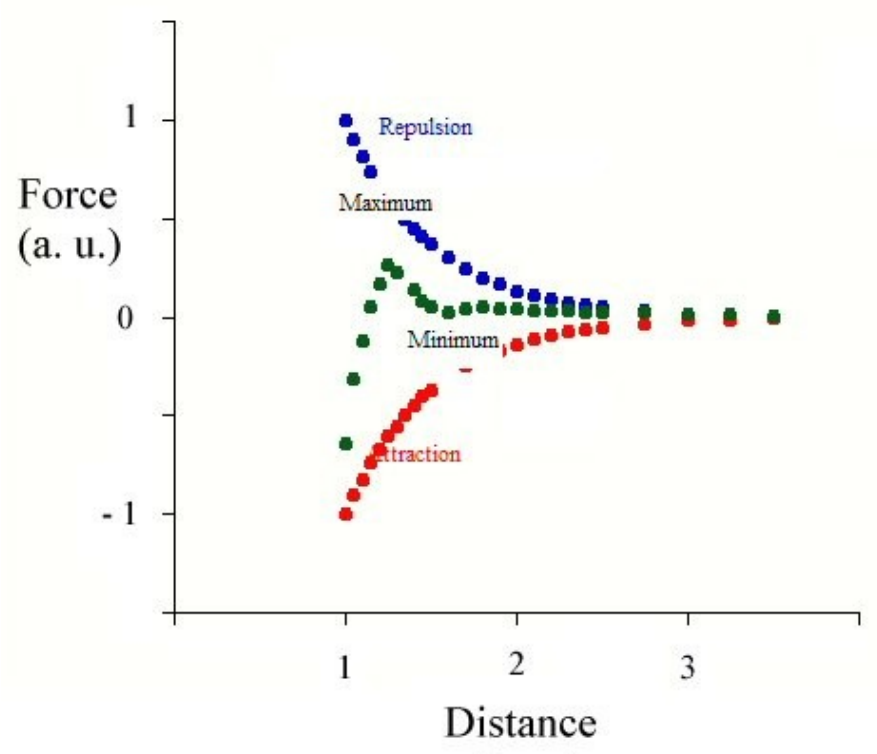

(a. u.)

Figure 5. Schematic view of the forces acting among two colloids, or surfaces, vs. distance. The plot refers to the region of interest, for distances slightly above $1 \mathrm{~nm}$. Repulsive (light blue), i.e. electrostatic, and attractive van der Waals (red) forces are considered. The result (in green) is the sum of these two contributions. Its inflection point refers to stability conditions, and its minimum corresponds to the minimum value Gibbs energy function. The position and amplitude of maxima and minima of the green curve depend on the medium ionic strength. 
There $\varrho$ is the ion, or charge, number density in the medium, $\varepsilon$ and $\varepsilon^{\circ}$ the permittivity of vacuum and of the dispersant, respectively. Since the electric field in $E q$. (5) is radial, we may consider its decay only along axis $x$, and rewrite it as

$$
\nabla 2 \Psi=d^{2} \Psi / d x^{2}=-\left(\varrho / \varepsilon \varepsilon^{\circ}\right)
$$

As to the statistical energy term, the Boltzmann's law for the distribution of charged species in a medium is used. It is written according to

$$
c_{i}=c_{i}{ }^{\circ} \exp -(z e \Psi / K B T)
$$

where $c_{i}$ is the local concentration of the $i^{\text {th }}$ ion, $c_{i}{ }^{\circ}$ its actual one, $z e \Psi$ the energy associated to the electric field for an ion of valence $z, K_{B} T$ the thermal energy. Eq. [6] is the ratio of electrical to thermal energy, and determines ion distribution around charged entities, depending on electric fields and thermal motions. Each ion contributes to the electric field, and

$$
\mathrm{Q}=\varepsilon\left(c^{+}-c^{-}\right)=\varepsilon c_{i}{ }^{\circ}\left(\exp ^{-\left(z e^{\Psi / K B T}\right)}-\exp ^{\left(z e \Psi^{\Psi} / K B T\right)}\right)
$$

In this form $E q .(8)$ represents the charge density due to ions in excess, either $>0$ or $<0$. And, if $\left|\left(z e \Psi / K_{B} T\right)\right|$ term is much less than unity, the difference of exponentials transforms in hyperbolic form $\left(\exp ^{x}-\exp ^{-x}=2 \sinh x\right)$, and can be later linearized. That form implies a "linear" perturbation regime, which is helpful in determining the electric contributions to Gibbs energy. The advantages inherent to such linearization procedures are, thus, substantial.

The charge density, $\varrho$, is related to the surface potential, $\sigma$, which also depends on $\Psi$. The links between $\varrho, \sigma$ and $\Psi$ are expressed as

$$
\begin{gathered}
\sigma=-\int \varrho d x \\
\sigma=\left(2 n^{\circ} \varepsilon K_{B} T / \Pi\right)^{1 / 2} \sinh \left(z e \Psi / K_{B} T\right)
\end{gathered}
$$

where the meaning of symbols is as before. From Eq. (9) and (10) we may relate part of the system energy to the corresponding electrical potentials, according to

$$
\Delta G_{e l}=-\int \sigma d \Psi
$$

Proper combination of electrical energy with all other ones allows analyzing experimental data and forecast the overall stabilization energy. 


\subsection{The fundamentals of food colloid stabilization}

Animal-based foods and our bodies contain about 55-75 wt $\%$ water, depending on the tissue. What remains is made of fats, lipids, sugars, proteins, associating in liquid, gel, liquid crystalline, paste, amorphous, or semi-solid matrices. Food tissues consistency depends on the organization of colloid entities in a tissue. From this evidence it comes out the generalization that animals, humans, and vegetables consist of different colloid entities, which, eventually, coagulate.

To show how much the overlapping of these effects is important in the food chain, we consider milk transformation. Although the process is well acquainted, the underlying theory is not yet fully applied. We do not consider transient stirring; in the above case, dispersions revert to the original conditions in the time lapse required to dissipate the mechanical energy imparted to the dispersions and later released in form of heat. Here focus is on "stable" food dispersions, having shelf-life of weeks or months. That is, on systems in thermodynamic, or kinetic, equilibrium conditions. Do not worry about the substantial physical difference among them, by the moment.

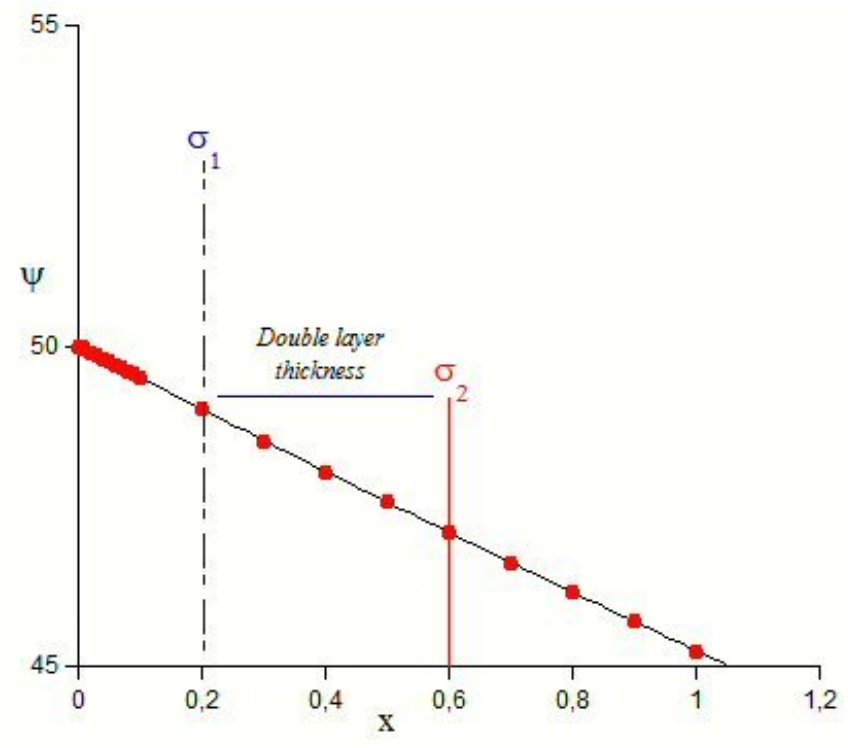

Figure 6. The decay of electrostatic potential, $\Psi$, versus distance, $x$. The distance among the points having charge density equal to $\sigma_{1}$ and $\sigma_{2}$ is the double layer thickness, or Debye's screening length, and depends on the medium ionic strength.

Coagulation among charged particles hardly occurs, because of repulsive electrostatic forces. This holds true in oil droplets stabilized by charged lipid layers. Even low amounts of electrolytes ensure droplets to repel each other. Conversely, if $\varrho, \sigma$, or $\Psi$, are small, the energy barrier among particles (always related to $z e \Psi$ ) is low, and a marked tendency to coagulation is observed. Thus, $D$ depends on the medium ionic strength, as is to be expected, and decreases with it; the above salting behavior is often used to reduce repulsive forces among similarly charged surfaces. The threshold at which such phenomena occur is the so-called flocculation limit. An increase of electrolyte decreases $\Psi$ and ensures coagulation. Thus, as $\sigma$ tends to $0, D L$ forces are null, electrostatic terms vanish, and the particles attract. When the $p H$, or the ionic strength, $I$, change food-based colloids aggregate, or re-disperse, depending on the experimental conditions. Steric stabilization, acting as an additional dispersive effect, overlaps with electrostatic forces and imparts to these dispersions a substantial stability, Figure 7. 


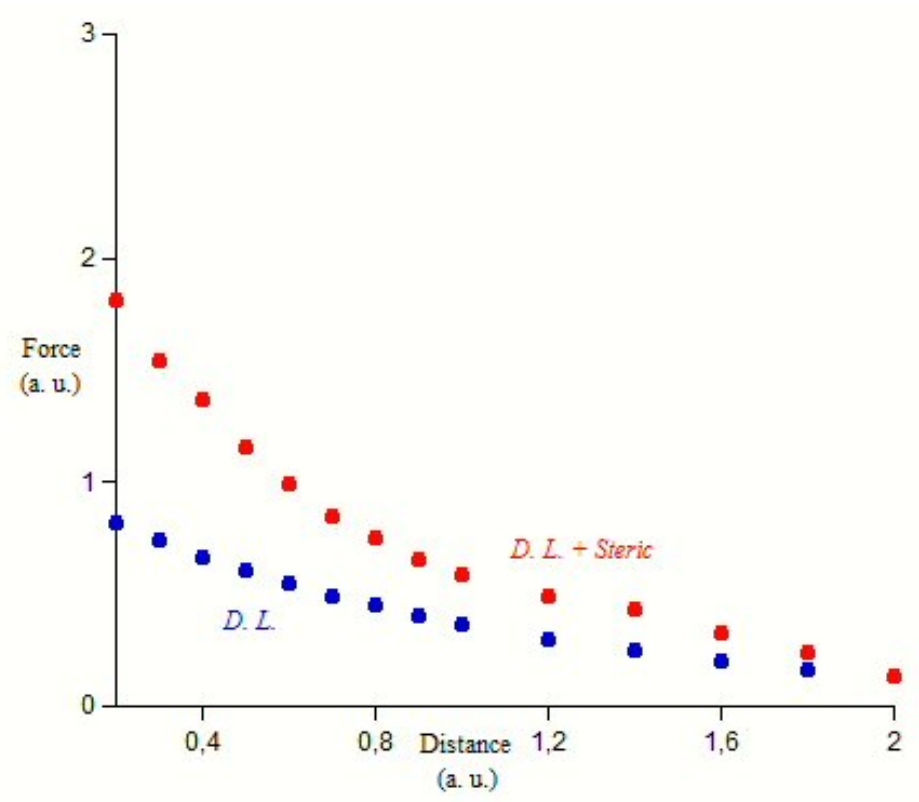

Figure 7. Overlapping of purely electrostatic terms, in blue, with steric stabilization, in red, forces at short distances. Forces are in arbitrary units. It is evident that elimination of steric terms partly reduces the system stability.

\subsection{The case of milk-based food colloids.}

Peculiar are milk manipulation methods, ending in cheese formation. The whole process is controlled by the relative amounts of fatty acids and glycerides in milk, both existing in the form of oil droplets, plus micelle-forming casein, proteolytic enzymes, salts, lactose (the main milk sugar component, later enzymatically transformed in lactic acid), and so forth. The process ends when aggregation/gelation occurs, is governed by $T$, enzymic activity, changes in $p H$, presence of salts, and combinations thereof. Many are the routes leading to coagulation, and forces may favor a seedlike or fibrous organization. This is why we get parmesan (with its hard to the teeth, and exhibits a typical "sandy" consistency) [46], provolone and mozzarella cheese (both consisting in more or less consistent fibrous structures) [47], or ricotta (soft, flaky, and easily spread). So large differences among the above products find origin in the fact that stretching, eventually combined with heating, deform cheese seeds and impart a preferred direction along which fibers orient by the action of cheese weight, or preferentially orient along the stress direction. Both stretching and subsequent deformations are somehow due to the plasticity of raw cheese paste. In consequence of that, they are different each from the other in terms of taste, smell, consistency, lifetime, and stiffness.

Although casein micelles are charged, added salt does not necessarily ensure coagulation, or formation of "cheese seeds". In fact, such micelle-like entities are stabilized by steric forces, not allowing them to come in contact and coagulate. Steric stabilization, thus, counteracts attractive forces. The effect is destroyed by rennet, a proteolytic enzyme cutting the $k$-casein parts facing outward micelles. In the early cheese-making steps of the procedure $p H$ values close to 4.8 activate/ deactivate the hydrolytic enzymes, whose activity also depends on $T$, and salts, eventually [48]. Sometimes, the above enzyme is replaced by twistle, or fig tree, branches, whose extracts have very pronounced proteolytic capacity. Rennet contains different enzymes, all produced in the stomachs of young ruminants; its key component, chimosin, is a protease, curdling milk casein; but also pepsin, sometimes phosphatases, and some lipases are present too.

Chimosin main action consists in the cleaving of $k$-casein chains [49]. Cleavage causes casein residues to stick to cleaved molecules of the like and to form a network. Rennet action, thus, 
a) eliminates the stabilizing moieties among casein micelles; and

b) makes possible to such entities to form a network clustering together fat droplets and some mineral colloids (usually phosphates). The latter entities are sometimes termed "cheese seeds". The structure of the resulting network depends on several variables. Size and elasticity of seeds depend on network size; the same holds for their consistency, aging modes and spreading facility (if any).

Rennet better clusters casein in presence of calcium and phosphate, occasionally added in the cheese making of goat milk, which is calcium phosphate-poor [50]. A solid-truncated casein protein network traps other components of milk, mostly fat droplets. Rennet also separates milk in solid curds (in the cheesemaking stages) and liquid whey, and so do its substitutes. Also relevant is the electrolyte concentration and valence; calcium, for instance, favors aggregation compared to both sodium or potassium. Thus, presence of ions and the subsequent screening of repulsive electrostatic forces are relevant, once the proteolytic activity has gone to completion. To clarify such aspects we introduce below a simple electrostatic approach to stabilization and show how much the latter is relevant in milk-based formulations.

As mentioned above, repulsive forces are electrical, steric, or osmotic. We consider first the role of the former in favoring/disfavoring phase separation. Steric, osmotic and $D L$ contributions counteract $v d W$ forces (which are ubiquitary); they shift the coagulation threshold to much higher concentrations. These features, observed in some stages of cheese making, are summarized below. In $D L V O$ theory, $v d W$ forces, which are of quantum mechanical origin, are ubiquitary. They are not always dominant, and combination with $D L$ ones gives rise to the overall system energy.

For two bodies at constant $T$ the interaction energy, $E_{\text {int }}$, depends of the reciprocal distance, $D$. At high $D$ 's $E_{\text {int }}$ and $D L$ terms approach zero; in such regime the sum of all contributions vanishes. No energy gain is associated to the interactions between particles at long distances, which remain in dispersed form. The presence of a primary minimum, at short distances, and of a secondary one are met when distances are much lower than those reported here. The secondary minimum shifts in proportion to $I$; it is separated from the primary one by an energy barrier, whose height depends on the activation energy required to get coagulation. The secondary minimum is some $K_{B} T$ units high and shifts to lower distances in proportion to $I$. The figure indicates that the tendency to coagulate is represented by the overlapping of energy curves with $v d W$ ones [51].

The process depends on the activation energy to coagulation, $E_{a t t}$. The role of ionic strength, $I$, $=1 / 2 \quad \sum_{\mathrm{i}=1} \mathrm{c}_{\mathrm{i}} \mathrm{Zi}^{2}$, is put in evidence by considering what happens when the potential among surfaces having a fixed number of charges per unit area is screened by due amounts of added salt. In distilled water, $\Psi^{\circ}$ rapidly increases with ion concentration. Neutral electrolytes (some $\mathrm{mmol} \mathrm{kg}^{-1}$ ) have a buffer effect on $\Psi^{\circ}$. Since most food preparations contain substantial amounts of salt, it is clear why the region were the potentials are effective is moderate and in the range $25-100 \mathrm{mV}$, in modulus. For $\Psi<125 \mathrm{IV}$, samples tend to coagulate; if $\Psi>1100 \mathrm{mV}$, conversely, most counter-ions adsorb on the particle surface and minimize repulsions. Thus, changes in $\Psi$ ensure dispersion, aggregation or sedimentation [52].

In the calculations we combine all the mentioned forces in the generalized relation

$$
E_{t o t}=\sum_{i=1} E_{i} \exp ^{-k i D}
$$

where $E_{i}$ is a given energy mode (be it $\mathrm{vdW}$, DL, or else). More intricate equations for the system energy scale with distance as $1 / D^{n}$ (with $n \geq 3$ ) and have been widely described [53]. These energy terms conform to short-distance decay modes. Thus, repulsion rapidly decreases with distance. The attractive terms, conversely, are mostly governed by $v d W$ terms, essentially of quantum-mechanical origin. They are responsible for phase separation. On this regard, the difference between the onset of yogurt and cheese coagulation clarifies what physical forces govern the onset of these materials.

Electrical potentials in colloids are measured by $\zeta$-potential a distance $d$ apart the outer limit of the slipping plane, by electrophoretic mobility or Laser-Doppler facility $[54,55]$. The decay of such 
$\zeta$-potentials with $p H$ and/or $I$ can be determined, and the surface charges may be partly titrated, with subsequent coagulation, or re-dispersion, Figure 8 . The salient point in the figure is the "nominal" point of zero charge, $p z c$. For proteins, the $p z c$ corresponds to their isoelectric point [56].

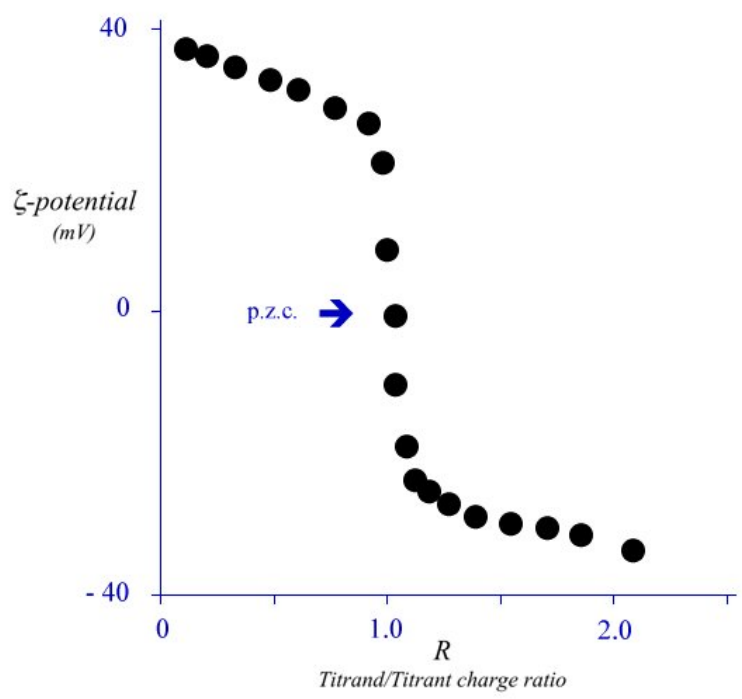

Figure 8. $\zeta$-potential, in $\mathrm{mV}$, vs versus the titrant to titrand, $R$, ratio. If $R \cong 1$, the $\zeta$ potential is 0 . The curve is grossly symmetrical with respect to the p.z.c. (potential of zero charge), the inflection point in the graph. Data reported therein refer to sunflower oil dispersed by $D P D C$, in 0.0250 aqueous $\mathrm{NaCl}$, at $35.0^{\circ} \mathrm{C}$. The titrand/titrant ratio, $R$, is related to the particle charge density, and depends on $p H$.

\subsection{The Cheese Making Sequence.}

Actual cheese-making sequences are not different from what historically known. They consist of 5 , sometimes 6 , stages. In turn:

1. Milk coagulation by rennet (sometimes assisted by tiny amounts of citric acid) added to milk kept at mild temperature, $36-39^{\circ} \mathrm{C}$. This step implies optimizing the enzymic activity of rennet and, in consequence of that, favors eliminating steric stabilization, by cutting the polypeptide chains protruding outward casein micelles.

2. Curd breaking and serum discharge jointly imply a phase separation of colloids, governed essentially by $v d W$ forces. A heating stage at $50^{\circ} \mathrm{C}$ is required, to complete discharge them and favor the nucleation of cheese lumps.

3. Curd is left maturing in acid serum for some hours. In this stage, solubilization of calcium salts and de-mineralization of cheese paste occur. The paste becomes plastic (is a reverse phase separation), giving rise to "pasta filata" (also termed cooked pasta), is reduced in fibers by the action of gravitation, or by simple stretching, as happens for mozzarella and provolone. In absence of shear forces, which favor the formation of fibers, cheese grains collapse and nucleate.

4. Salting is made in dry or wet conditions, depending on the needs. Ion valence and content is relevant in this step. Salting implies water loss, due to the onset of osmotic gradients outside the cheese peel, which contracts, deforms, and progressively becomes harder.

5. Cheese maturing, usually performed at $3-8{ }^{\circ} \mathrm{C}$ and $85-90 \%$ humidity. It helps giving cheese the due consistency and appearance. It implies a further water loss, with seeds packing. Maturing depends on the cheese kind to be obtained, and the same holds for the working conditions. In niche products mildewing, coverage with straw, or chestnut leaves (which 
are rich in tannins), is sometimes used. Sometimes, mildewed conditions are induced, on the cheese peel or in its interior. In the latter eventuality, a wide variety of "blue cheese" types may occur. These can be relatively stiff (Stilton), or creamy (as soft Gorgonzola).

\section{Conclusions}

The transformation of raw foods that can be properly stored, is well known since the very early stages of human civilization. It is urgent, nowadays, to optimize these transformations in such a way that the products obtained by any food manipulation chain are safe and can be stored for long times. That is the reason why efforts are currently done in the search to optimize the properties of primary matter and subsequent formulations in real food. To do this, advanced scientific approaches must supplement what is done in common-use procedures. The above efforts actually find considerable attention, also due to the fact that the amount of available food resources is not high if compared to the number of humans worldwide. Transformation of primary matter, thus, requires to be optimized in such a way to get food(s) having good nutritional quality, safety and substantial attitudes to be stored even in non-optimal conditions. To proceed long this line, we rely on an incontrovertible fact. Most primary raw matter is usually transformed in products having colloid nature. The latter take the form of pastes, creams, solids, and semi-solid matter, whose physical consistency spans from that peculiar to yogurt to that of seasoned Parmesan cheese.

Theoretical approaches based on the fundamentals of colloid chemistry, thus, find a rationale. By them we can evaluate the role of the forces mentioned above in determining optimal sequences and hierarchy of the procedures giving rise to a given colloid organization mode. In doing this we operate exactly in the same way the was historically used in optimizing a given product compared to others. The only substantial difference to old-fashioned prepares is that we may control each step.

We are aware of the fact that the contributions to the overall system stability due to the forces indicated before are substantially different, in modulus, each from the other. In addition, some of them are always attractive (as $v d W$ ), other repulsive (as $D L$ ones). Evaluation of all these effects can, thus, be cumbersome. What is more, the effects due to each of them depends on distance, that is on the concentration of the dispersed matter, and scales according to different power law modes. Think, for instance, to the substantial difference among the aforementioned $v d W$ and $D L$ forces!

It is also important to check if it is possible to pass, and in which way, from finely dispersed to a coagulated state, as experimentally observed in some stages of the food manipulation. These aspects are relevant in forecasting experimental results, and check if they are well modulated.

The calculus procedures and methodological approaches suggested here are made possible only by considering the mentioned terms in a sort of hierarchical scale, that is by progressively adding one more term of those of minor relevance. This procedure will help defining the real contributions pertinent to each of the energy modes that are present in any food preparation chain.

We regret if our technical knowledge of main effective cheese production chains is limited to the role of appraisers and knowers. That is why we essentially focused on the forces in action for each milk manipulation step, without entering into much technical details on the complex art termed cheese-making. Being not capable to enter into technical details, we conform to the disconsolate opinion that a French Politician of the last century, Charles De Gaulle, used when retired from the active policy. He said "It is impossible to govern a country worldwide famous for producing well over 400 different cheese kinds."

Author Contributions: The authors jointly contributed to the manuscript. GR took care of the biological-biochemical parts; CLM dealt with the physicochemical bases. Writing, review and editing were jointly made by both authors.

Funding: This research received no external funding. 
Acknowledgments: CLM wishes to acknowledge a dear friend, Giuseppe Ranieri, formerly at Calabria University, for providing copy of an old-dated article by Chandrasekkhar, Ref. [1], and Lorenza Suber, formerly at CNR, for suggesting Arbasino's book, cited in Ref. [2].

Conflicts of Interest: The authors declare no conflict of interest. 


\section{References}

1. S. Chandrasekkhar, Stochastic Problems in Physics and Astronomy. Rev. Mod. Phys., (1943), 15, 1. [N.B. His mathematical treatment of stellar evolution later gave rise to many theoretical models on the early evolutionary stages of massive stars and black holes. And on colloids too, mostly on theories of diffusion in porous media.]

2. A. Arbasino, Un paese senza, Garzanti, Milano, (1980), 40. [N.B. The late Alberto Arbasino passed over in March 2020 at the age of 90 . He was a brilliant and argumentative writer, and a sarcastic polemicist. We translated this piece, regretting not to have been fully capable to transmit its temper. Pinocchio has possibly a world-wide resonance, and the same holds for Le Chat Botteè. The Red Feathered Young Teacher may be less known. However, she is a main character in a short story of the book "Cuore" a popular reading for primary school children (but not only them) by the Italian XIX century novelist, painter, and poet Edmondo De Amicis.]

3. S. Mukhopadhyay, Nano Drugs: A Critical Review of Their Patents and Market. In "Characterization and Biology of Nanomaterials for Drug Delivery”, Elsevier, Amsterdam, (2019), Chapt. 18, 523-551.

4. M.A.J.S. van Boekel; W.M.F. Jongen Product quality and food processing: how to quantify the healthiness of a product. Cancer Lett., (1997), 114, 65-69.

5. M. Toussaint-Samat “A History of Food", Wiley, New York (2009).

6. D. M.Post The long and short of food-chain length. Trends Ecology and Evolution, (2002), 117, 269-277.

7. S.I. Martínez-Guido; J.B. González-Campos; M.M. El-Halwagi; J.M. Ponce-Ortega. Sustainable Optimization of Food Networks in Disenfranchised Communities. ACS Sustainable Chem. Eng., (2017), 5, 8895-8907.

8. S.Rea; G. Storani; N. Mascaro; R. Stocchi; A.R. Loschi. Species identification in anchovy pastes from the market by PCR-RFLP technique. Food Control, (2009), 20, 515-520.

9. T.P. Guinee; B.T. O’Kennedy; P.M. Kelly. Effect of Milk Protein Standardization Using Different Methods on the Composition and Yields of Cheddar Cheese. J. Dairy Sci., (2006), 89, 468-482.

10. Y. Zhong; G. Cavender; Y. Zhao. Investigation of different coating application methods on the performance of edible coatings on Mozzarella cheese. LWT - Food Sci. Technol., (2014), 56, 1-8.

11. M.P. Longinotti; M.F. Mazzobre; M.P. Buera; H.R. Corti. Effect of salts on the properties of aqueous sugar systems in relation to biomaterial stabilization. Part 2: Sugar crystallization rate and electrical conductivity behavior. PCCP, (2002), 4, 533-540.

12. M.E. Leser; L. Sagalowicz; M. Michel; H.J. Watzke. Self-assembly of polar food lipids. Adv. Colloid Interface Sci., (2006), 123,125-136.

13. B.S. Murray; K. Durga; A. Yusoff; S.D. Stoyanov. Stabilization of foams and emulsions by mixtures of surface active food-grade particles and proteins. Food Hydrocolloids, (2011), 25, 627-638.

14. C. Schmitt; S.L.Turgeon. Protein/polysaccharide complexes and coacervates in food systems. Adv. Colloid Interface Sci., (2011), 167, 63-70.

15. T. Eftimov; P. Korošec; B. Koroušić Seljak. StandFood: Standardization of Foods Using a Semi-Automatic System for Classifying and Describing Foods According to FoodEx2. Nutrients (2017), 9, 542-558.

16. Milk Proteins From Expression to Food. Boland M., Singh, H., Ed.s; 3rd Ed. Acad. Press, (2019).

17. G. Getaneh; A. Mebrat; A. Wubie; H. Kendie. Review on Goat Milk Composition and its Nutritive Value. J. Nutr. Health Sci., (2016), 3, 1-10.

18. H. Schott. Saturation adsorption at interfaces of surfactant solutions. J. Pharm. Sci., (1980), 69, 852-854.

19. S.R. Accordino; M.A. Morini; M.B. Sierra; J.A. Rodríguez Fris; G.A. Appignanesi; A. Fernández. Wrapping mimicking in drug-like small molecules disruptive of protein-protein interfaces. Proteins, (2012), 80, 1755-1765.

20. L. Mohrhusen; M. Osmić. Steric ligand stabilization of nanocrystals versus electrostatic shielding by ionic compounds: a principle model study with TEM and XPS. RSC Adv., (2017), 7, 12897-12907.

21. H.N.W. Lekkerkerker. Osmotic Equilibrium Treatment of the Phase Separation in Colloidal Dispersions Containing Non-Adsorbing Polymer Molecules. Colloids Surf., (1990) 51, 419-426.

22. C. Schneider; M. Hanisch; B. Wedel; A. Jusufi; M. Ballauff. Experimental study of electrostatically stabilized colloidal particles: Colloidal stability and charge reversal. J. Colloid Interface Sci., (2011), 358, 62-67.

23. Th. F. Tadros. Interfacial Phenomena and Colloid Stability: Industrial Applications. (2015), Wiley.

24. A.I. Usov, Polysaccharides of the red algae. Adv. Carbohydrate Chem. Biochem., (2011), 65, 115- 217.

25. A.G. Marangoni, J.P. M. van Duynhoven, N.C. Acevedo, R.A. Nicholson, A.R. Patel. Advances in our understanding of the structure and functionality of edible fats and fat mimetics. Soft Matter, (2020), 16, 289-306.

26. E. Dickinson Stability and rheological implications of electrostatic milk protein-polysaccharide interactions. Trends Food Sci. Technol., (1998), 9, 347-354. 
27. N. Yaranossian; M.G. Lynch, Confectionery containing low viscosity iota carrageenan. EP3197287A2, (2019), European Patent.

28. D.J. Finney; I.C.M. Dea. Stabilization of ice-cream compositions. CA1109319A (1992), Canadian Patent.

29. D.H. Everett. Basic Principles of Colloid Science. Roy. Soc. Chem., (1994), Chaps. V, 63-75.

30. R.H. Ottewill; C.H. Rochester; A.L. Smith, Ed.s. Adsorption from Solution. Acad. Press, London, (1983).

31. K. Lunkenheimer; K.-D. Wantke. Determination of the surface tension of surfactant solutions applying the method of Lecomte du Noüy (ring tensiometer). Colloid Polym. Sci., (1981), 259, 354-366.

32. V.S.Alahverdjieva; D.O. Grigoriev; J.K. Ferri; V.B. Fainerman; E.V. Aksenenko; M.E. Leser; M. Michel; R. Miller. Adsorption behavior of hen egg-white lysozyme at the air/water interface. Colloids Surf. A, Physicochem. Engin. Aspects, (2008) 323, 167-174. See also Chapt. III in Ref. [51].

33. S. Louguet; A.C. Kumar; N. Guidolin; D. Signed; E. Duguet; S. Lecommandoux; C. Shatz. Control of the PEO Chain Conformation on Nanoparticles by Adsorption of PEO-block-Poly(L-lysine) Copolymers and Its Significance on Colloidal Stability and Protein Repellency. Langmuir, (2011), 27, 12891-12901.

34. X. Yuan Hua; M.J. Rosen. Dynamic surface tension of aqueous surfactant solutions: I. Basic parameters. J. Colloid Interface Sci., (1988), 124, 652-659.

35. A. Bonfillon; F. Sicoli; D. Langevin. Dynamic Surface Tension of Ionic Surfactant Solutions. J. Colloid Interface Sci., (1994), 168, 497-504.

36. F. Rouquerol; J. Rouquerol; K.S.W. Sing; P. Llewellyn, G. Maurin. Adsorption by Powders and Porous Solids. Principles, Methodology and Applications. Acad. Press. (2013).

37. P.G. De Gennes. Scaling theory of polymer adsorption. J. Phys., France, (1976), 37, 1445-1452.

38. D. Hone; H. Ji; P. A. Pincus. Polymer Adsorption on Rough Surfaces, 1. Ideal Long Chain. Macromolecules, (1987), 20, 2543-2549.

39. R. Evans; D.H. Napper. Steric stabilization I. Comparison of theories with experiment. Koll. Z.u.Z. Polym., (1973), 251, 409-414. [N.B. The authors gave the first systematic approach to explain on solid grounds the concept of steric stabilization.]

40. J. Israelachvili; Y. Min; M. Akbulut; A Alig; G Carver; W. Greene; K. Kristiansen; E. Meyer; N. Pesika; K. Rosenberg; H. Zeng. Recent advances in the surface forces apparatus (SFA) technique. Rep. Prog. Phys., (2010), 73, 036601 (16pp).

41. E.B. Zhulina; O.V. Borisov; V. A. Priamitsyn. Theory of steric stabilization of colloid dispersions by grafted polymers. J. Colloid Interface Sci., (1990), 137, 495-511.

42. L.D. Landau, L.D. Collected Works. Nauka (Moscow), (1969), Vol. I, 386-411.

43. E.J.W. Verwey; J.Th.G. Overbeek. Theory of the Stability of Lyophobic Colloids. Dover Books on Chemistry, (1999). [N.B. Is the reprint of the 1948 original version of this seminal book.]

44. R.V. Deriaguin. Theory of the Stability of Colloids and Thin Films. Consultants Bureau, New York and London, (1989).

45. B.W. Ninham. On progress in forces since DLVO theory. Adv. Colloid Interface Sci., (1999), 83, 1-17.

46. Look adjective articles in the Cookie Master column, by J. Moskin, regularly appearing in The New York Times. That column is extremely rich of information on unknown and puzzling aspects of food sciences (and not only of recipes). A very pertinent one dates to Nov. 7, 2004.

47. "The cheese that melts and stretches", in "Italian Food Tech", 11 October 2012.

48. Manufacturing method for fibrous cheese products. JP2017510252A, Japan Patent, (2014).

49. C. Beumer; M. Wulferink; W. Raaben; D. Fiechter; R. Brands; W. Seinen. Calf Intestinal Alkaline Phosphatase, a Novel Therapeutic Drug for Lipopolysaccharide (LPS)-Mediated Diseases, Attenuates LPS Toxicity in Mice and Piglets. J. Pharmacol. Exp. Therapeutics, (2003), 307, 737-744.

50. G.S. Manning. Counterion Condensation on Charged Spheres, Cylinders and Planes. J. Phys. Chem. B, (2007), 111, 8554-8559.

51. A.I. Nájera; M.de Renobales; L.J.R. Barrona. Effects of $\mathrm{pH}$, temperature, $\mathrm{CaCl}_{2}$ and enzyme concentrations on the rennet-clotting properties of milk: a multifactorial study. Food Chemistry, (2003), 80, 345-352.

52. C. Carles; B. Ribadeau Dumas. Kinetics of the action of chymosin (rennin) on a peptide bond of bovine $\alpha s_{1}$-casein: Comparison of the behavior of this substrate with that of $\beta$ - and xo-caseins. FEBS Lett., (1985), 185, 282-286.

53. A. Montilla; M.M. Calvo. Goat's Milk Stability during Heat Treatment: Effect of pH and Phosphates. J. Agric. Food Chem. (1997), 45, 931934.

54. J.N. Israelachvili. Intermolecular and Surface Forces. Elsevier, III' ${ }^{\mathrm{rd}}$ Ed., (2011).

55. A.V. Adamson. Physical Chemistry of Surfaces. Vth Ed.; Wiley, (1991), Chapt. V. 
56. H. Ohshima. Electrophoretic mobility of soft particles. Colloids and Surfaces A: Physicochemical and Engineering Aspects, (1995), 103, 249-255.

57. D.G. Dalgleish. Measurement of electrophoretic mobilities and zeta-potentials of particles from milk using laser Doppler electrophoresis. J. Dairy Res., (1984), 51, 425-438.

58. K.A. Kantardjieff; B. Rupp. Protein isoelectric point as a predictor for increased crystallization screening efficiency. Bioinformatics, (2004), 20, 2162-2168. 